\title{
GENERALIZED EULER CONSTANTS FOR ARITHMETICAL PROGRESSIONS
}

\author{
KARL DILCHER
}

\begin{abstract}
The work of Lehmer and Briggs on Euler constants in arithmetical progressions is extended to the generalized Euler constants that arise in the Laurent expansion of $\zeta(s)$ about $s=1$. The results are applied to the summation of several classes of slowly converging series. A table of the constants is provided.
\end{abstract}

\section{INTRODUCTION}

The Riemann zeta function $\zeta(s)$ has the following Laurent expansion about its pole $s=1$ :

$$
\zeta(s)=\frac{1}{s-1}+\sum_{k=0}^{\infty} A_{k}(s-1)^{k} .
$$

The coefficients $A_{k}$ are given by

$$
A_{k}=\frac{(-1)^{k}}{k !} \gamma_{k}
$$

where

$$
\gamma_{k}=\lim _{n \rightarrow \infty}\left\{\sum_{j=1}^{n} \frac{\log ^{k} j}{j}-\frac{\log ^{k+1} n}{k+1}\right\}, \quad k=0,1,2, \ldots
$$

(we set $\log ^{0} 1=1$ ). It is clear that $\gamma_{0}=\gamma$, the Euler constant. The expansion (1.1) was independently established by several authors, and the $\gamma_{k}$ have been studied quite extensively. For a short historical survey, see [3, p. $164 \mathrm{f}$.]; more recent references not included in [3] are $[4,11,17,18,19]$. The coefficients $A_{k}$ are usually called Stieltjes constants, and the numbers $\gamma_{k}$ are sometimes referred to as generalized Euler constants.

In this paper we shall consider generalizations of $\gamma_{k}$ that arise by taking the sum in (1.2) over an arithmetical progression. More exactly, denote

$$
H_{k}(x, r, m)=\sum_{\substack{0<n \leq x \\ n \equiv r(\bmod m)}} \frac{\log ^{k} n}{n},
$$

Received by the editor December 6, 1990.

1991 Mathematics Subject Classification. Primary 11M06; Secondary 11Y60, 65B10, 65B15. 
and define

$$
\gamma_{k}(r, m):=\lim _{x \rightarrow \infty}\left\{H_{k}(x, r, m)-\frac{\log ^{k+1} x}{m(k+1)}\right\} .
$$

These constants were previously studied by Knopfmacher [14] as special cases of a wider class of generalized Euler constants. The case $k=0$ was considered by Briggs [6] and Lehmer [15].

The purpose of this paper is to derive further properties of the constants $\gamma_{k}(r, m)$, several of them generalizing results in $[6,15]$. This is done in $\S \S 2-$ 4 . In $\S 5$ (and already earlier) this will be applied to the summation of several classes of very slowly converging series; some of these results are known, while others appear to be new. In $\S 6$ we shall derive explicit expressions for $\gamma_{1}(r, m)$, $m=3,4$, and 6 . A connection with a class of generalized gamma functions will be given in $\S 7$. Finally, in $\S 8$ we shall make some remarks on numerical calculations of the $\gamma_{k}(r, m)$; a table is provided, with $1 \leq k \leq 20,1 \leq m \leq 9$, and $1 \leq r \leq m$.

\section{BASIC PROPERTIES OF THE $\gamma_{k}(r, m)$}

The existence of the limit in (1.4) was deduced by Knopfmacher as an application of his more general theory in [14]. This and other results from [14] could also be proved directly by following the corresponding proofs for $k=0$ in [15].

It follows immediately from (1.3) and (1.4) that

$$
\gamma_{k}(1,1)=\gamma_{k}
$$

and, since $H_{k}(x, r \pm m, m)=H_{k}(x, r, m)$, we have

$$
\gamma_{k}(r \pm m, m)=\gamma_{k}(r, m) \text {. }
$$

Because of (2.2), we may restrict our attention to $1 \leq r \leq m$.

Proposition 1 [14]. Let $k \geq 0, m \geq 1$, and let $d$ be a common divisor of $r$ and $m$. Then

$$
\gamma_{k}(r, m)=\frac{1}{d} \sum_{\nu=0}^{k}\left(\begin{array}{l}
k \\
\nu
\end{array}\right)(\log d)^{k-\nu} \gamma_{\nu}\left(\frac{r}{d}, \frac{m}{d}\right)-\frac{\log ^{k+1} d}{m(k+1)} .
$$

If we set $r=d=m$ and take (2.1) into account, we get the following special case of (2.3).

Corollary 1. For all $k \geq 0$ and $m \geq 1$ we have

$$
\gamma_{k}(m, m)=\frac{1}{m} \sum_{\nu=0}^{k}\left(\begin{array}{l}
k \\
\nu
\end{array}\right)(\log m)^{k-\nu} \gamma_{\nu}-\frac{\log ^{k+1} m}{(k+1) m}
$$

In particular,

$$
\begin{aligned}
& \gamma_{0}(m, m)=\frac{1}{m}(\gamma-\log m), \\
& \gamma_{1}(m, m)=\frac{1}{m}\left(\gamma_{1}+\gamma \log m-\frac{1}{2} \log ^{2} m\right) .
\end{aligned}
$$


Note that with $k=0$ in $(2.3)$ we get

$$
\gamma_{0}(r, m)=\frac{1}{d} \gamma_{0}\left(\frac{r}{d}, \frac{m}{d}\right)-\frac{1}{m} \log d ;
$$

this is just Theorem 2 in [15]. Also, (2.5) is equation (2) in [15]. The next property is another analogue to a result (containing a misprint) in [15]; it is almost immediate from (1.4) and (1.3).

Proposition 2. There holds

$$
\sum_{j=0}^{n-1} \gamma_{k}(r+j m, n m)=\gamma_{k}(r, m) .
$$

In particular,

$$
\sum_{r=1}^{m} \gamma_{k}(r, m)=\gamma_{k} .
$$

Proposition 3. For $k \geq 0$ and $m \geq 1$ we have

$$
\begin{aligned}
\gamma_{k}(m, 2 m)= & \frac{1}{2 m} \sum_{\nu=0}^{k}\left(\begin{array}{l}
k \\
\nu
\end{array}\right) \gamma_{\nu}\left(2 \log ^{k-\nu} m-\log ^{k-\nu} 2 m\right) \\
& -\frac{2 \log ^{k+1} m-\log ^{k+1} 2 m}{2 m(k+1)} .
\end{aligned}
$$

In particular,

$$
\begin{aligned}
& \gamma_{0}(m, 2 m)=\frac{1}{2 m}\left(\gamma-\log \frac{m}{2}\right), \\
& \gamma_{1}(m, 2 m)=\frac{1}{2 m}\left(\gamma_{1}+\gamma \log \frac{m}{2}+\frac{1}{2} \log ^{2} 2 m-\log ^{2} m\right) .
\end{aligned}
$$

Proof. Equation (2.7) with $n=2$ and $r=0$ gives

$$
\gamma_{k}(m, 2 m)=\gamma_{k}(0, m)-\gamma_{k}(0,2 m)=\gamma_{k}(m, m)-\gamma_{k}(2 m, 2 m) \text {. }
$$

The assertion (2.9) now follows from (2.4).

\section{SERIES REPRESENTATIONS FOR $\gamma_{k}(r, m)$}

We define the arithmetic function $g_{r}^{(m)}(n)$ by

$$
g_{r}^{(m)}(n)= \begin{cases}m-1 & \text { when } n \equiv r(\bmod m), \\ -1 & \text { otherwise. }\end{cases}
$$

Proposition 4. For $k \geq 0, m \geq 2$, and $0 \leq r \leq m-1$ we have

$$
m \gamma_{k}(r, m)=\gamma_{k}+\sum_{n=1}^{\infty} \frac{\log ^{k} n}{n} g_{r}^{(m)}(n) .
$$

Proof. Following [15], we denote for $0 \leq j \leq m-1$,

$$
\sigma_{j}:=\sum_{\lambda=0}^{m-1} \gamma_{k}(\lambda, m) e^{2 \pi i \lambda j / m} .
$$


Then by $(2.8)$,

$$
\sigma_{0}=\gamma_{k}
$$

To simplify notation, we write $\varepsilon:=\exp (2 \pi i / m)$. Let $j \neq 0$. We use the fact that

$$
\sum_{\lambda=0}^{m-1} \varepsilon^{\lambda j}=0
$$

Then by (1.4) and (1.3) we have

$$
\sigma_{j}=\lim _{x \rightarrow \infty}\left\{\sum_{\lambda=0}^{m-1}\left(H_{k}(x, \lambda, m)-\frac{\log ^{k+1} x}{(k+1) m}\right) \varepsilon^{\lambda j}\right\},
$$

that is,

$$
\sigma_{j}=\sum_{n=1}^{\infty} \frac{\log ^{k} n}{n} \varepsilon^{n j}
$$

We solve (3.2) for the $\gamma_{k}(\lambda, m)$ by multiplying both sides by $\varepsilon^{-j r}$ and summing over $j$. Taking (3.4) into account, we get

$$
\sum_{j=0}^{m-1} \sigma_{j} \varepsilon^{-j r}=\sum_{\lambda=0}^{m-1} \gamma_{k}(\lambda, m) \sum_{j=0}^{m-1} \varepsilon^{j(\lambda-r)}=m \gamma_{k}(r, m) .
$$

Hence, with (3.3) and (3.5) we get

$$
m \gamma_{k}(r, m)=\gamma_{k}+\sum_{j=1}^{m-1} \varepsilon^{-j r} \sum_{n=1}^{\infty} \frac{\log ^{k} n}{n}-\varepsilon^{n j}
$$

Now formally,

$$
m \gamma_{k}(r, m)=\gamma_{k}+\sum_{n=1}^{\infty} \frac{\log ^{k} n}{n} \sum_{j=1}^{m-1} \varepsilon^{j(n-r)} .
$$

The change in the order of summation of this conditionally convergent series can be justified by taking the appropriate limits for $\sigma_{j}$ and the infinite series. Now by the definition of $g_{r}^{(m)}(n)$ and by (3.4) we have

$$
\sum_{j=1}^{m-1} \varepsilon^{j(n-r)}=g_{r}^{(m)}(n)
$$

The result (3.1) now follows from (3.7).

Remark. For an easier proof of (3.1), see the example after Proposition 9. However, we still require (3.6) and (3.7) elsewhere.

Proposition 5. For $k \geq 0$ and $m \geq 2$ we have

$$
\sum_{n=1}^{\infty} \frac{\log ^{k} n}{n} g_{0}^{(m)}(n)=\sum_{\nu=0}^{k-1}\left(\begin{array}{l}
k \\
\nu
\end{array}\right)(\log m)^{k-\nu} \gamma_{\nu}-\frac{\log ^{k+1} m}{k+1}
$$

Proof. Take $r=0$ in (3.1) and combine with (2.3).

We consider now the special cases $k=1$ and (independently) $m=2$. If we note that $g_{0}^{(2)}(n)=(-1)^{n}$, the following becomes obvious from (3.8). 
Corollary 2. For $m \geq 2$ we have

$$
\gamma=\frac{1}{2} \log m+\frac{1}{\log m} \sum_{n=2}^{\infty} \frac{\log n}{n} g_{0}^{(m)}(n)
$$

and in particular

$$
\gamma=\frac{1}{2} \log 2+\frac{1}{\log 2} \sum_{n=2}^{\infty}(-1)^{n} \frac{\log n}{n}
$$

For $k \geq 0$ we have

$$
\sum_{n=1}^{\infty}(-1)^{n} \frac{\log ^{k} n}{n}=\sum_{\nu=0}^{k-1}\left(\begin{array}{l}
k \\
\nu
\end{array}\right)(\log 2)^{k-\nu} \gamma_{\nu}-\frac{\log ^{k+1} 2}{k+1} .
$$

Corollary 3. If we set

$$
\tau_{k}^{(m)}:=\sum_{n=1}^{\infty} \frac{\log ^{k} n}{n} g_{0}^{(m)}(n), \quad \tau_{0}(m)=-\log m,
$$

then we have for all $m \geq 2$

$$
\gamma_{k}=\frac{1}{k+1} \sum_{j=0}^{k+1}\left(\begin{array}{c}
k+1 \\
j
\end{array}\right)(\log m)^{j-1} B_{j} \tau_{k+1-j}^{(m)},
$$

where $B_{j}$ is the jth Bernoulli number.

Proof. This was shown in [16] for $m=2$ by inverting the matrix of the triangular linear system (3.11). The proof carries over to arbitrary $m$ without change, solving the linear system (3.8).

Remarks. 1. While (3.10) is a well-known series expansion for the Euler constant (see, e.g., [10, p. 288]), (3.9) appears to be little known. It was previously proved by Kluyver [13] and rediscovered by Jacobsthal [12].

2. The expression (3.12) was proved by Kluyver [13]; note that a different notation for the Bernoulli numbers was used in [13].

3. The expansion (3.11) was proved by Liang and Todd [16]; it was used there to compute the $\tau_{k}=\tau_{k}^{(2)}$ for $1 \leq k \leq 20$ to 15 digits accuracy from the previously computed $\gamma_{k}$.

4. With $k=0$ in (3.8) we get

$$
\begin{aligned}
\log m= & -\sum_{n=1}^{\infty} \frac{1}{n} g_{0}^{(m)}(n) \\
= & 1+\frac{1}{2}+\cdots+\frac{1}{m-1}-\frac{m-1}{m}+\frac{1}{m+1} \\
& +\cdots+\frac{1}{2 m-1}-\frac{m-1}{2 m}+\cdots ;
\end{aligned}
$$

this was proved in $[15$, p. $136 ; 12]$; it is a generalization of the alternating harmonic series for $\log 2$.

Next we replace $m$ by $2 m$ in (3.1) and set $r=m$. Then we get the following result. 
Proposition 6. For $k \geq 0$ and $m \geq 1$ we have

$$
\begin{aligned}
\sum_{n=1}^{\infty} \frac{\log ^{k} n}{n} g_{m}^{(2 m)}(n)= & \sum_{\nu=0}^{k-1}\left(\begin{array}{l}
k \\
\nu
\end{array}\right)\left(2 \log ^{k-\nu} m-\log ^{k-\nu} 2 m\right) \gamma_{k} \\
& -\frac{1}{k+1}\left(2 \log ^{k+1} m-\log ^{k+1} 2 m\right) .
\end{aligned}
$$

Again, we consider special cases, for $k=0,1,2$ and for $m=2$.

Corollary 4. For $m \geq 1$ we have

$$
\sum_{n=1}^{\infty} \frac{1}{n} g_{m}^{(2 m)}(n)=-\log \frac{m}{2}
$$

$$
\sum_{n=2}^{\infty} \frac{\log n}{n} g_{m}^{(2 m)}(n)=\gamma \log \frac{m}{2}-\log ^{2} m+\frac{1}{2} \log ^{2} 2 m,
$$

and in particular

$$
\begin{aligned}
& \sum_{n=2}^{\infty} \frac{\log n}{n} g_{2}^{(4)}(n)=\log ^{2} 2 \\
& \sum_{n=2}^{\infty} \frac{\log ^{2} n}{n} g_{m}^{(2 m)}(n)= \gamma\left(2 \log ^{2} m-\log ^{2} 2 m\right)+2 \gamma_{1}(2 \log m-\log 2 m) \\
&-\frac{2}{3} \log ^{3} m+\frac{1}{3} \log ^{3} 2 m,
\end{aligned}
$$

and in particular

$$
\gamma=\log 2-\frac{1}{2 \log 2} \sum_{n=2}^{\infty} \frac{\log ^{2} n}{n} g_{2}^{(4)}(n) .
$$

Remarks. 1. With $m=1$ in (3.14) we get (3.11) again; note that $g_{1}^{(2)}(n)=$ $(-1)^{n-1}$.

2. Formula (3.15) is another generalization of the type (3.13) of the alternating harmonic series for $\log 2$. For $m=2$ we get

$$
-1+\frac{3}{2}-\frac{1}{3}-\frac{1}{4}-\frac{1}{5}+\frac{3}{6}-\frac{1}{7}-\frac{1}{8}-\frac{1}{9}+\frac{3}{10}-\frac{1}{11}-\cdots=0 .
$$

\section{Primitive $\gamma_{k}(r, m)$}

Following Lehmer [15], we call $\gamma_{k}(r, m)$ primitive when $r$ and $m$ are coprime. If $\gamma_{k}(r, m)$ is not primitive, then it can be expressed, via (2.3), in terms of primitive $\gamma_{\nu}\left(r_{1}, m_{1}\right), 0 \leq \nu \leq k$. This suggests a more detailed study of primitive $\gamma_{k}(r, m)$.

In agreement with the notation in [15], we set

$$
\phi_{k}(m):=\sum_{\substack{(r, m)=1 \\ 1 \leq r \leq m}} \gamma_{k}(r, m)
$$


For $j=0,1,2, \ldots$ we introduce the sums

$$
N_{j}(m):=\sum_{d \mid m} \mu(d) \frac{m}{d} \log ^{j} d,
$$

where $\mu(d)$ is the Möbius function. In particular, we have

$$
N_{0}(m)=\sum_{d \mid m} \mu(d)=\varphi(m)
$$

(see, e.g., [2, p. 26]) and

$$
N_{1}(m)=-\varphi(m) \sum_{p \mid m} \frac{\log p}{p-1}
$$

(see $[15$, p. 131$]$ ), where $\varphi(m)$ is the Euler totient function. The following result is a generalization of [15, Theorems 3-5].

Proposition 7. For all $k \geq 0$ we have

$$
m \phi_{k}(m)=\sum_{j=0}^{k}\left(\begin{array}{l}
k \\
j
\end{array}\right) \gamma_{j} N_{k-j}(m)-\frac{1}{k+1} N_{k+1}(m) .
$$

Proposition 7 could be proved by induction, following the proof of Theorem 3 in [15]. However it is easier to proceed as follows. We first need a lemma.

Lemma 1. For all $k \geq 0$ and $m \geq 1$ we have

$$
\sum_{(r, m)=1} \gamma_{k}(r, m)=\sum_{d \mid m} \mu(d) \gamma_{k}(0, d) .
$$

Proof. We use (2.7) with $r=0, m=d$, and $n=m / d$, to get

$$
\sum_{j=0}^{m / d-1} \gamma_{k}(j d, m)=\gamma_{k}(0, d)=\gamma_{k}(d, d) .
$$

Multiplying both sides by $\mu(d)$ and summing over all $d \mid m$, we obtain

$$
\begin{aligned}
\sum_{d \mid m} \mu(d) \gamma_{k}(d, d) & =\sum_{d \mid m} \mu(d) \sum_{j=0}^{m / d-1} \gamma_{k}(j d, m) \\
& =\sum_{r=0}^{m-1} \gamma_{k}(r, m) \sum_{d \mid(r, m)} \mu(d) .
\end{aligned}
$$

Here the inner sum was obtained by noting that $d$ has to divide both $r$ and $m$, hence the g.c.d. of $r$ and $m$. By a basic property of the Möbius function (see, e.g., [2, p. 25]) the inner sum is zero unless $(r, m)=1$. Thus we obtain the left-hand side of (4.6), and the proof is complete.

Example. Let $m=6$ and $k=0$. Then (4.6) becomes

$$
\gamma(1,6)+\gamma(5,6)=\gamma(1,1)-\gamma(2,2)-\gamma(3,3)+\gamma(6,6) .
$$

This agrees with Table 1 in [15]. 
Proof of Proposition 7. In (2.4) we replace $m$ by $d$, multiply both sides by $\mu(d)$, and sum over all $d \mid m$. Then, by (4.1) and (4.6), we have

$$
m \phi_{k}(m)=\sum_{d \mid m} \mu(d) \frac{m}{d}\left\{\sum_{\nu=0}^{k}\left(\begin{array}{l}
k \\
\nu
\end{array}\right)(\log d)^{k-\nu} \gamma_{\nu}-\frac{\log ^{k+1} d}{k+1}\right\} .
$$

If we now change the order of summation and use (4.2), we get the right-hand side of (4.5). This completes the proof.

Remarks. 1. With $k=0$ in (4.5), and using (4.3) and (4.4), we get

$$
m \phi_{0}(m)=\varphi(m)\left\{\gamma+\sum_{p \mid m} \frac{\log p}{p-1}\right\} .
$$

This is Theorem 3 combined with Theorem 5 in [15]; see also [6].

2. With $m=1,(4.5)$ reduces to $(2.1)$.

3. Knopfmacher [14] proved the following version of (4.5). For prime numbers $p$, define

$$
c_{0}(p):=1-\frac{1}{p} \quad \text { and } \quad c_{i}(p):=(-1)^{i+1} \frac{\log ^{i} p}{p} \quad(i \geq 1) .
$$

Now for integers $m \geq 1$ denote

$$
a_{j}^{(m)}:=\sum \prod_{p \mid m} c_{i_{p}}(p)
$$

where the sum is taken over all $i_{p}$ with $\sum_{p} i_{p}=j$. Then, in our notation,

$$
\frac{(-1)^{k}}{k !} \phi_{k}(m)=a_{k+1}^{(m)}+\sum_{j=0}^{k} \frac{k !}{j !}(-1)^{k-j} \gamma_{j} a_{k-j}^{(m)} .
$$

Incidentally, comparing (4.5) and (4.7), we can prove by induction (beginning with $\left.a_{0}^{(m)}=N_{0}(m) / m\right)$ that

$$
N_{j}(m)=(-1)^{j} m j ! a_{j}^{(m)} .
$$

Lehmer showed in [15] that if $\delta$ is a divisor of $m$, then $\phi_{0}(\delta m)=\phi_{0}(m)$. This property holds also for $\phi_{k}(m), k \geq 1$.

Corollary 5. Let $k \geq 0, m \geq 1$, and $\delta \mid m$. Then $\phi_{k}(\delta m)=\phi_{k}(m)$.

Proof. Any divisor $d$ of $\delta m$ that is not already a divisor of $m$ has to be divisible by a square. But $\mu(d)=0$ if $d$ is not square free. Hence, by (4.2) we have $N_{j}(\delta m)=\delta N_{j}(m)$ for $j=0,1,2, \ldots$. The result follows from (4.5).

The next application of Proposition 7 is an analogue of Theorem 6 in [15].

Corollary 6. For $k \geq 0$ and $m \geq 2$ we have

$$
\begin{gathered}
\sum_{j=1}^{m-1}\left(\sum_{n=1}^{\infty} \frac{\log ^{k} n}{n} \cos \frac{2 \pi n j}{m}\right) \varphi(m) \frac{\mu(m /(m, j))}{\varphi(m /(m, j))} \\
=\sum_{j=0}^{k-1}\left(\begin{array}{l}
k \\
j
\end{array}\right) \gamma_{j} N_{k-j}(m)-\frac{N_{k+1}(m)}{k+1} .
\end{gathered}
$$


Proof. We sum (3.6) over all $r<m$ relatively prime to $m$. Then, with (4.1), we get

$$
m \phi_{k}(m)=\varphi(m) \gamma_{k}+\sum_{j=1}^{m-1}\left(\sum_{n=1}^{\infty} \frac{\log ^{k} n}{n} \varepsilon^{n j}\right) \sum_{(r, m)=1} e^{-2 \pi i j r / m} .
$$

The last sum in (4.9) is a Ramanujan sum $c_{m}(j)$, evaluated by Hölder's formula

$$
c_{m}(j)=\varphi(m) \frac{\mu(m /(m, j))}{\varphi(m /(m, j))}
$$

(see, e.g., [2, p. 164]). Since all the other terms in (4.9) are real, we may replace the infinite sum by its real part. We get now (4.8) by applying (4.5).

Remark. If in (4.8) we let $m$ be a prime $p$ or a power of a prime, we will get (3.8) for $m=p$. To see this, we use the fact that

$$
\sum_{j=0}^{m-1} \cos \frac{2 \pi r j}{m}=0 \quad \text { if } r \equiv 0(\bmod m)
$$

and apply the definition of $g_{r}^{(m)}(n)$. Also note that with (4.2) we have for integers $\alpha$,

$$
N_{j}\left(p^{\alpha}\right)=p^{\alpha-1} \log ^{j} p, \quad j=0,1,2, \ldots
$$

If $m$ has at least two different prime factors, (4.8) will give new summation formulas. However, it will be more convenient to use (3.1) for this purpose.

Corollary 7. For $k \geq 0$ and $m \geq 2$ we have

$$
\sum_{n=1}^{\infty} \frac{\log ^{k} n}{n} \sum_{(m, r)=1} g_{r}^{(m)}(n)=\sum_{j=0}^{k-1}\left(\begin{array}{c}
k \\
j
\end{array}\right) \gamma_{j} N_{k-j}(m)-\frac{1}{k+1} N_{k+1}(m)
$$

and in particular

$$
\begin{gathered}
\sum_{n=1}^{\infty} \frac{1}{n} \sum_{(m, r)=1} g_{r}^{(m)}(n)=\varphi(m) \sum_{p \mid m} \frac{\log p}{p-1}, \\
\sum_{n=1}^{\infty} \frac{\log n}{n} \sum_{(m, r)=1} g_{r}^{(m)}(n) \\
=-\gamma \varphi(m) \sum_{p \mid m} \frac{\log p}{p-1}-\frac{1}{2} \sum_{d \mid m} \mu(d) \frac{m}{d} \log ^{2} d .
\end{gathered}
$$

Proof. Sum both sides of (3.1) over all $r<m$ relatively prime to $m$, and apply (4.1) and (4.5). The change in the order of summation can be justified by going back to the limits defining the $\gamma_{k}(r, m)$.

Example. Let $m=6$, the smallest positive integer with two different prime divisors. Let

$$
h^{(6)}(n)=\sum_{(6, r)=1} g_{r}^{(6)}(m)=g_{1}^{(6)}(n)+g_{5}^{(6)}(n)= \begin{cases}4 & \text { if } n \equiv 1,5(\bmod 6) \\ -2 & \text { otherwise }\end{cases}
$$


Then, with (4.11) and (4.12), we get respectively

$$
\begin{gathered}
\sum_{n=1}^{\infty} \frac{1}{n} h^{(6)}(n)=2 \log 2+\log 3 \\
\sum_{n=1}^{\infty} \frac{\log n}{n} h^{(6)}(n)=(\log 3-2 \log 2) \gamma+\frac{1}{2}\left(3 \log ^{2} 2+2 \log ^{2} 3-\log ^{2} 6\right) .
\end{gathered}
$$

We note that if $h^{(m)}(n)$ is defined as above, we always have

$$
h^{(m)}(n)= \begin{cases}m-\varphi(m) & \text { if }(n, m)=1, \\ -\varphi(m) & \text { if }(n, m)>1\end{cases}
$$

\section{SUMMATION OF CERTAIN SERIES}

In this section we consider two applications of the $\gamma_{k}(r, m)$ to the summation of series involving $k$ th powers of the logarithm. The first application is an analogue to Theorem 9 in [15].

Proposition 8. Let $k \geq 0, N \geq 2$, and let $\left(r_{1}, m_{1}\right),\left(r_{2}, m_{2}\right), \ldots,\left(r_{N}, m_{N}\right)$ be pairs of positive integers for which $1 \leq r_{j} \leq m_{j}, j=1,2, \ldots, N$, and such that the rational numbers $r_{j} / m_{j}$ are distinct. Then the series

$$
S_{k}:=\sum_{n=0}^{\infty}\left\{c_{1} \frac{\log ^{k}\left(m_{1} n+r_{1}\right)}{m_{1} n+r_{1}}+\cdots+c_{N} \frac{\log ^{k}\left(m_{N} n+r_{N}\right)}{m_{N} n+r_{N}}\right\}
$$

converges if and only if

$$
\sum_{j=1}^{N} \frac{c_{j}}{m_{j}} \log ^{\nu} m_{j}=0 \text { for } \nu=0,1, \ldots, k
$$

If the series (5.1) converges, then

$$
S_{k}=\sum_{j=1}^{N} c_{j}\left\{\gamma_{k}\left(r_{j}, m_{j}\right)+\frac{1}{m_{j}} \frac{\log ^{k+1} m_{j}}{k+1}\right\} .
$$

Proof. We adapt the proof of Theorem 9 in [15]. We have

$$
\begin{aligned}
S_{k}= & \lim _{x \rightarrow \infty} \sum_{j=1}^{N} c_{j} \sum_{0 \leq n \leq x} \frac{\log ^{k}\left(m_{j} n+r_{j}\right)}{m_{j} n+r_{j}} \\
= & \lim _{x \rightarrow \infty} \sum_{j=1}^{N} c_{j} H_{k}\left(m_{j} x, r_{j}, m_{j}\right) \\
= & \sum_{j=1}^{N} c_{j} \lim _{x \rightarrow \infty}\left\{H_{k}\left(m_{j} x, r_{j}, m_{j}\right)-\frac{\log ^{k+1}\left(m_{j} x\right)}{(k+1) m_{j}}\right\} \\
& +\lim _{x \rightarrow \infty} \sum_{j=1}^{N} \frac{c_{j}}{m_{j}} \frac{\log ^{k+1}\left(m_{j} x\right)}{k+1} .
\end{aligned}
$$


Now we use (1.4), and in the second sum we expand $\log ^{k+1}\left(m_{j} x\right)$ with the binomial theorem. Then, after a change in the order of summation, we get

$$
\begin{aligned}
S_{k}= & \sum_{j=1}^{N} c_{j} \gamma_{k}\left(r_{j}, m_{j}\right)+\sum_{j=1}^{N} \frac{c_{j}}{m_{j}} \frac{\log ^{k+1} m_{j}}{k+1} \\
& +\lim _{x \rightarrow \infty}\left\{\sum_{\nu=0}^{k} \frac{1}{k+1}\left(\begin{array}{c}
k+1 \\
\nu
\end{array}\right)\left(\sum_{j=1}^{N} \frac{c_{j}}{m_{j}} \log ^{\nu} m_{j}\right) \log ^{k+1-\nu} x\right\} .
\end{aligned}
$$

It is now clear that the limit exists if and only if (5.2) holds for $\nu=0,1, \ldots, k$. This proves Proposition 8.

Example. Let

$$
S_{1}:=\sum_{n=0}^{\infty}\left\{\frac{\log (n+1)}{n+1}-2 \frac{\log (2 n+1)}{2 n+1}+\frac{\log 2}{2 n+1}\right\}
$$

We can rewrite

$$
S_{1}=\sum_{n=0}^{\infty}\left\{\frac{\log (n+1)}{n+1}-4 \frac{\log (2 n+1)}{2 n+1}+4 \frac{\log (4 n+2)}{4 n+2}\right\}
$$

and verify that (5.2) holds. Hence the series converges, and we have by (5.3),

$$
S_{1}=\gamma_{1}(1,1)-4\left\{\gamma_{1}(1,2)+\frac{1}{2} \frac{\log ^{2} 2}{2}\right\}+4\left\{\gamma_{1}(2,4)+\frac{1}{4} \frac{\log ^{2} 4}{2}\right\} \text {. }
$$

Finally, with (2.1) and (2.11), we get

$$
S_{1}=(3 \log 2+2 \gamma) \log 2 \text {. }
$$

We consider now the special case where all the $m_{j}$ are equal. Then (5.2) reduces to the condition that the sum of the $c_{j}$ be zero, and consequently the logarithmic terms in (5.3) disappear. Hence we have

Corollary 8. Let $k \geq 0, N \geq 2$, and let $m$ and $r_{1}, r_{2}, \ldots, r_{N}$ be positive integers such that $0<r_{1}<r_{2}<\cdots<r_{N} \leq m$. Let $c_{1}, c_{2}, \ldots, c_{N}$ be real or complex numbers such that $c_{1}+c_{2}+\cdots+c_{N}=0$. Then

$$
\sum_{n=0}^{\infty}\left\{c_{1} \frac{\log ^{k}\left(m n+r_{1}\right)}{m n+r_{1}}+\cdots+c_{N} \frac{\log ^{k}\left(m n+r_{N}\right)}{m n+r_{N}}\right\}=\sum_{j=1}^{N} c_{j} \gamma_{k}\left(r_{j}, m\right) .
$$

In two particular cases, the sum of the series can be given in terms of the $\gamma_{j}$, $j=0,1, \ldots, k$.

Corollary 9. For $k \geq 0$ and $m \geq 2$ we have

$$
\begin{gathered}
\sum_{n=0}^{\infty}\left\{\sum_{j=1}^{m-1} \frac{\log ^{k}(m n+j)}{m n+j}-(m-1) \frac{\log ^{k}(m(n+1))}{m(n+1)}\right\} \\
=-\sum_{\nu=0}^{k-1}\left(\begin{array}{l}
k \\
\nu
\end{array}\right)(\log m)^{k-\nu} \gamma_{\nu}+\frac{\log ^{k+1} m}{k+1}
\end{gathered}
$$


Proof. We use (5.4) with $c_{1}=c_{2}=\cdots=c_{m-1}=1, c_{m}=1-m$. With (2.8) and (2.4), the right-hand side of (5.4) becomes

$$
\begin{gathered}
\sum_{j=1}^{m-1} \gamma_{k}(j, m)-(m-1) \gamma_{k}(m, m)=\sum_{j=1}^{m} \gamma_{k}(j, m)-m \gamma_{k}(m, m) \\
=\gamma_{k}-\sum_{\nu=0}^{k}\left(\begin{array}{l}
k \\
\nu
\end{array}\right)(\log m)^{k-\nu} \gamma_{\nu}+\frac{1}{k+1} \log ^{k+1} m .
\end{gathered}
$$

This proves (5.5), since the terms involving $\gamma_{k}$ are cancelled.

Example. For $k=1$, the right-hand side of $(5.5)$ is simply $((\log m) / 2-\gamma) \log m$.

Corollary 10. For $k \geq 0$ and even integers $m \geq 4$ we have

$$
\begin{aligned}
\sum_{n=0}^{\infty}\left\{\sum_{(j, m)=1} \frac{\log ^{k}(m n+j)}{m n+j}-\varphi(m) \frac{\log ^{k}(m n+m / 2)}{m n+m / 2}\right\} \\
=\frac{1}{m} \sum_{j=0}^{k}\left(\begin{array}{c}
k \\
j
\end{array}\right) \gamma_{j}\left\{N_{k-j}(m)-\varphi(m)\left(2 \log ^{k-j} \frac{m}{2}-\log ^{k-j} m\right)\right\} \\
-\frac{1}{m(k+1)}\left\{N_{k+1}(m)-\varphi(m)\left(2 \log ^{k+1} \frac{m}{2}-\log ^{k+1} m\right)\right\},
\end{aligned}
$$

where the $N_{j}(m)$ are defined by (4.2). In particular,

$$
\begin{aligned}
\sum_{n=0}^{\infty}\left\{\sum_{(j, m)=1} \frac{\log (m n+j)}{m n+j}-\varphi(m) \frac{\log (m n+m / 2)}{m n+m / 2}\right\} \\
=-\gamma \frac{\varphi(m)}{m}\left\{\sum_{p \mid m} \frac{\log p}{p-1}+\log m-2 \log 2\right\} \\
-\frac{1}{2 m}\left\{\sum_{d \mid m} \mu(d) \frac{m}{d} \log ^{2} d-\varphi(m)\left(2 \log ^{2}\left(\frac{m}{2}\right)-\log ^{2} m\right)\right\} .
\end{aligned}
$$

Proof. We apply (5.4) with $N=m, r_{j}=j$ for $j=1,2, \ldots, m, c_{j}=1$ when $(j, m)=1, c_{j}=0$ otherwise except $c_{m / 2}=-\varphi(m)$. Then the right-hand side of (5.4) becomes $\phi_{k}(m)-\varphi(m) \gamma_{k}(m / 2, m)$, where $\phi_{k}(m)$ is defined by (4.1). We obtain now the right-hand side of (5.6) from (4.5) and (2.9). With (4.4), we immediately get (5.7).

Example. With $m=6$, the relation (5.7) becomes after some calculation

$$
\sum_{n=0}^{\infty}\left\{\frac{\log (6 n+1)}{6 n+1}-2 \frac{\log (6 n+3)}{6 n+3}+\frac{\log (6 n+5)}{6 n+5}\right\}=\frac{\log 3}{2}\left(\frac{1}{2}-\log 2-\gamma\right) .
$$

The second application of the $\gamma_{k}(r, m)$ is an analogue of Theorem 8 in [15], proved by Knopfmacher [14]. 
Proposition 9 [14]. Let $g(n)$ be an arithmetical function, periodic with period $m$. Then

$$
S_{k}(g):=\sum_{n=1}^{\infty} g(n) \frac{\log ^{k} n}{n}
$$

converges if and only if

$$
\sum_{j=1}^{m} g(j)=0
$$

If $S_{k}(g)$ converges, then

$$
S_{k}(g)=\sum_{r=1}^{m} g(r) \gamma_{k}(r, m) .
$$

Example. The function $g(n)=g_{r}^{(m)}(n)$, as defined in $\S 3$, satisfies (5.9). Hence, Proposition 9 implies (3.1) upon applying (2.8).

A second application of Proposition 9 is given in the next section.

\section{SOME EXPLICIT EXPRESSIONS}

Let $g(n)=\chi(n)$ be a nonprincipal Dirichlet character modulo $m$, and

$$
L(s, \chi)=\sum_{n=1}^{\infty} \frac{\chi(n)}{n^{s}}
$$

the corresponding Dirichlet $L$-series. Then its $k$ th derivative is

$$
L^{(k)}(s, \chi)=(-1)^{k} \sum_{n=1}^{\infty} \chi(n) \frac{\log ^{k} n}{n^{s}},
$$

and with Proposition 9 we get the following, as was noted by Knopfmacher [14].

Proposition 10 [14]. For $k \geq 0$ we have

$$
L^{(k)}(1, \chi)=(-1)^{k} \sum_{r=1}^{m} \chi(r) \gamma_{k}(r, m) .
$$

For $k=0$, this was shown by Lehmer [15]. The connection between $L(1, \chi)$ and the class number of quadratic fields enabled Lehmer in [15] to give explicit expressions for the right-hand side of (6.1) in the case $k=0$.

If $k=1$ and $\chi$ is an odd character (i.e., $\chi(-1)=-\chi(1)$ ) modulo $f$, then $L^{\prime}(1, \chi)$ can be expressed in terms of classical functions (see, e.g., [8, p. 182]),

$$
L^{\prime}(1, \chi)=i \pi \frac{\tau(\chi)}{f}\left\{(\gamma+\log 2 \pi) B_{1, \bar{\chi}}+\sum_{\nu=1}^{f} \bar{\chi}(\nu) \log \Gamma\left(\frac{\nu}{f}\right)\right\},
$$

where

$$
\tau(\chi)=\sum_{\nu=0}^{f} \chi(\nu) e^{2 \pi i \nu / f}
$$


is the normalized Gauss sum associated with $\chi$, and $B_{1, \chi}$ is the first generalized Bernoulli number belonging to the character $\chi$; it can be expressed as

$$
B_{1, \chi}=\frac{1}{f} \sum_{\nu=1}^{f} \chi(\nu) \nu .
$$

In the special case where $\chi$ is an odd quadratic character, we have $\bar{\chi}=\chi$ and $\tau(\chi)=i \sqrt{f}$ (see, e.g., [5, p. 349]). Also, since $\chi(f-\nu)=-\chi(\nu)$, and using the well-known functional equation $\Gamma(1-z) \Gamma(z)=\pi / \sin \pi z$, we have

$$
\begin{aligned}
\sum_{\nu=1}^{f} \chi(\nu) \log \Gamma\left(\frac{\nu}{f}\right) & =\sum_{\nu=1}^{[f / 2]} \chi(\nu) \log \frac{\Gamma(\nu / f)}{\Gamma((f-\nu) / f)} \\
& =\sum_{\nu=1}^{[f / 2]} \chi(\nu) \log \left(\Gamma\left(\frac{\nu}{f}\right)^{2} \frac{\sin (\pi \nu / f)}{\pi}\right) .
\end{aligned}
$$

Hence, with (6.2), we get

$$
\begin{aligned}
L^{\prime}(1, \chi)=-\frac{\pi}{\sqrt{f}}\left\{\begin{array}{l}
(\gamma \\
+
\end{array}\right. & \log 2 \pi) B_{1, \chi} \\
& \left.+\sum_{\nu=1}^{[f / 2]} \chi(\nu) \log \left[\Gamma\left(\frac{\nu}{f}\right)^{2} \frac{\sin (\pi \nu / f)}{\pi}\right]\right\} .
\end{aligned}
$$

Example 1. Let $f=3$, i.e., $\chi(1)=1, \chi(2)=-1, \chi(3)=0$. Then from (6.4) we have $B_{1, \chi}=-\frac{1}{3}$, and we get with $(6.5)$

$$
L^{\prime}(1, \chi)=-\frac{\pi}{\sqrt{3}}\left\{(\gamma+\log 2 \pi) \frac{-1}{3}+\log \left[\Gamma\left(\frac{1}{3}\right)^{2} \frac{\sqrt{3}}{2 \pi}\right]\right\} .
$$

Now by (6.1) we have

$$
\gamma_{1}(1,3)-\gamma_{1}(2,3)=-L^{\prime}(1, \chi) .
$$

On the other hand, (2.6) gives

$$
\gamma_{1}(0,3)=\gamma_{1}(3,3)=\frac{1}{3}\left(\gamma_{1}+\gamma \log 3-\frac{1}{2} \log ^{2} 3\right),
$$

and with (2.8) we have

$$
\gamma_{1}(1,3)+\gamma_{1}(2,3)=\gamma_{1}-\gamma_{1}(0,3)=\frac{2}{3} \gamma_{1}-\frac{1}{3} \gamma \log 3+\frac{1}{6} \log ^{2} 3 .
$$

Finally, we combine (6.6) and (6.7) to get

$$
\begin{aligned}
& \gamma_{1}(1,3)=\frac{1}{2}\left\{\frac{2}{3} \gamma_{1}-\frac{1}{3} \gamma \log 3+\frac{1}{6} \log ^{2} 3-L^{\prime}(1, \chi)\right\}, \\
& \gamma_{1}(2,3)=\frac{1}{2}\left\{\frac{2}{3} \gamma_{1}-\frac{1}{3} \gamma \log 3+\frac{1}{6} \log ^{2} 3+L^{\prime}(1, \chi)\right\} .
\end{aligned}
$$

Example 2. Let $f=4$, i.e., $\chi(1)=1, \chi(3)=-1, \chi(2)=\chi(4)=0$. Then (6.4) gives $B_{1, \chi}=-\frac{1}{2}$, and (6.5) gives

$$
L^{\prime}(1, \chi)=\frac{-\pi}{2}\left\{(\gamma+\log 2 \pi) \frac{-1}{2}+\log \left[\Gamma\left(\frac{1}{4}\right)^{2} \frac{\sqrt{2}}{2 \pi}\right]\right\} \text {. }
$$


Now by $(6.1)$ we have

$$
\gamma_{1}(1,4)-\gamma_{1}(3,4)=-L^{\prime}(1, \chi) .
$$

From (2.6) and (2.11) we get

$$
\begin{aligned}
& \gamma_{1}(2,4)=\frac{1}{4}\left(\gamma_{1}+\log ^{2} 2\right), \\
& \gamma_{1}(4,4)=\frac{1}{4}\left(\gamma_{1}+2 \gamma \log 2-2 \log ^{2} 2\right),
\end{aligned}
$$

and (2.8) gives

$$
\begin{aligned}
\gamma_{1}(1,4)+\gamma_{1}(3,4) & =\gamma_{1}-\gamma_{1}(2,4)-\gamma_{1}(4,4) \\
& =\frac{1}{2} \gamma_{1}-\frac{1}{2} \gamma \log 2+\frac{1}{4} \log ^{2} 2 .
\end{aligned}
$$

We combine (6.10) and (6.11) to get

$$
\begin{aligned}
& \gamma_{1}(1,4)=\frac{1}{2}\left\{\frac{1}{2} \gamma_{1}-\frac{1}{2} \gamma \log 2+\frac{1}{4} \log ^{2} 2-L^{\prime}(1, \chi)\right\}, \\
& \gamma_{1}(3,4)=\frac{1}{2}\left\{\frac{1}{2} \gamma_{1}-\frac{1}{2} \gamma \log 2+\frac{1}{4} \log ^{2} 2+L^{\prime}(1, \chi)\right\} .
\end{aligned}
$$

Example 3. Let $f=6$, i.e., $\chi(1)=1, \chi(5)=-1, \chi(n)=0$ for all other $n$ $(\bmod 6)$. Note that the character $\chi$ is not primitive; we can write

$$
L(s, \chi)=\left(1+2^{-s}\right) L(s, \psi),
$$

where $\psi$ is the quadratic character of conductor 3 from Example 1. Differentiating both sides, we get

$$
L^{\prime}(1, \chi)=\frac{3}{2} L^{\prime}(1, \psi)-\frac{\log 2}{2} L(1, \psi) .
$$

To determine $L(1, \psi)$, we use the well-known generalized Euler formula

$$
L(1, \psi)=i \pi \frac{\tau(\psi)}{f} B_{1, \psi}
$$

(see, e.g., [8, p. 182]). With $\tau(\psi)=i \sqrt{f}, f=3$, and $B_{1, \psi}=-\frac{1}{3}$, this becomes $L(1, \psi)=\sqrt{3} \pi / 9$, so that

$$
L^{\prime}(1, \chi)=\frac{3}{2} L^{\prime}(1, \psi)-\frac{\log 2}{18} \sqrt{3} \pi .
$$

With (2.3), we have now for $r=1$ and $r=2$,

$$
\gamma_{1}(2 r, 6)=\frac{\log 2}{2} \gamma_{0}(r, 3)+\frac{1}{2} \gamma_{1}(r, 3)-\frac{1}{12} \log ^{2} 2 \text {. }
$$

In [15] we find, with $\varepsilon(1)=1, \varepsilon(2)=-1$,

$$
\gamma_{0}(r, 3)=\frac{1}{3} \gamma+\varepsilon(r) \frac{\pi}{18} \sqrt{3}+\frac{1}{6} \log 3,
$$

and with (6.8) and (6.9) we get

$$
\begin{aligned}
\gamma_{1}(2,6)= & \frac{\log 2}{6}\left(\gamma+\frac{\pi}{6} \sqrt{3}+\frac{\log 3}{2}\right) \\
& +\frac{1}{12}\left(2 \gamma_{1}-\gamma \log 3+\frac{1}{2} \log ^{2} 3-\log ^{2} 2\right)-\frac{1}{4} L^{\prime}(1, \psi),
\end{aligned}
$$




$$
\begin{aligned}
\gamma_{1}(4,6)= & \frac{\log 2}{6}\left(\gamma-\frac{\pi}{6} \sqrt{3}+\frac{1}{2} \log 3\right) \\
& +\frac{1}{12}\left(2 \gamma_{1}-\gamma \log 3+\frac{1}{2} \log ^{2} 3-\log ^{2} 2\right)+\frac{1}{4} L^{\prime}(1, \psi) .
\end{aligned}
$$

Also, by (2.11) and (2.6) we have

$$
\begin{aligned}
& \gamma_{1}(3,6)=\frac{1}{6}\left(\gamma_{1}+\gamma \log \frac{3}{2}+\frac{1}{2} \log ^{2} 6-\log ^{2} 3\right), \\
& \gamma_{1}(6,6)=\frac{1}{6}\left(\gamma_{1}+\gamma \log 6-\frac{1}{2} \log ^{2} 6\right) .
\end{aligned}
$$

Now, with (2.8) and (6.13)-(6.16), we get

$$
\begin{aligned}
& \gamma_{1}(1,6)+\gamma_{1}(5,6)= \gamma_{1}-\left[\gamma_{1}(2,6)+\gamma_{1}(4,6)\right]-[ \\
&=\frac{1}{3}\left\{\gamma_{1}-\gamma(3,6)+\gamma_{1}(6,6)\right] \\
&\left.+\frac{1}{2} \log ^{2} 2+\frac{1}{4} \log ^{2} 3\right\} .
\end{aligned}
$$

On the other hand, by (6.1) and (6.12), we have

$$
\gamma_{1}(1,6)-\gamma_{1}(5,6)=-\frac{3}{2} L^{\prime}(1, \psi)+\frac{\log 2}{18} \sqrt{3} \pi,
$$

and combining this with (6.17), we obtain

$$
\begin{aligned}
\gamma_{1}(1,6)= & \frac{1}{6}\left\{\gamma_{1}-\gamma\left(\log 2+\frac{1}{2} \log 3\right)-\frac{1}{2} \log 2 \log 3+\frac{1}{2} \log ^{2} 2+\frac{1}{4} \log ^{2} 3\right\} \\
& -\frac{3}{4} L^{\prime}(1, \psi)+\frac{\log 2}{36} \sqrt{3} \pi, \\
\gamma_{1}(5,6)= & \frac{1}{6}\left\{\gamma_{1}-\gamma\left(\log 2+\frac{1}{2} \log 3\right)-\frac{1}{2} \log 2 \log 3+\frac{1}{2} \log ^{2} 2+\frac{1}{4} \log ^{2} 3\right\} \\
& +\frac{3}{4} L^{\prime}(1, \psi)-\frac{\log 2}{36} \sqrt{3} \pi .
\end{aligned}
$$

Finally, we note that the numerical values of the explicit expressions in Examples 1-3 agree with the computed values in Table 1.

\section{CONNECTIONS WITH GENERALIZED GAMMA FUNCTIONS}

Let $\psi(z)$ be the logarithmic derivative $\psi(z)=\Gamma^{\prime}(z) / \Gamma(z)$ of the gamma function. Lehmer [15, p. 133] proved the following relationship between $\psi(z)$ and $\gamma_{0}(r, m)$ :

$$
\gamma_{0}(r, m)=-\frac{1}{m}\left\{\psi\left(\frac{r}{m}\right)+\log m\right\} .
$$

In this section we shall prove an analogue of (7.1) involving $\gamma_{k}(r, m)$ for arbitrary $k \geq 0$. For this purpose, we introduce higher analogues of the $\psi$-function as follows. For integers $k \geq 0$ and for complex $z \neq 0,-1,-2, \ldots$ let

$$
\psi_{k}(z):=-\gamma_{k}-\frac{\log ^{k} z}{z}-\sum_{\nu=1}^{\infty}\left\{\frac{\log ^{k}(\nu+z)}{\nu+z}-\frac{\log ^{k} \nu}{\nu}\right\} .
$$

Note that for $k=0$ this is a well-known representation for $\psi(z)$. For general $k$, this function occurs in Ramanujan's second notebook (see [3, Chapter 8, 
Entry 22]). The case $k=1$ was studied by Deninger [8]. The function $\psi_{k}(z)$ can be considered as the logarithmic derivative of an analogue $\Gamma_{k}(z)$ of the gamma function; for properties of this $\Gamma_{k}(z)$ and further connections with the $\gamma_{k}$, see [9].

Proposition 11. For $k \geq 0, m \geq 1$, and $1 \leq r \leq m$ we have

$$
\gamma_{k}(r, m)=\frac{-1}{m}\left\{\frac{\log ^{k+1} m}{k+1}+\sum_{\nu=0}^{k}\left(\begin{array}{l}
k \\
\nu
\end{array}\right)(\log m)^{k-\nu} \psi_{\nu}\left(\frac{r}{m}\right)\right\} .
$$

In particular, for $k=0$ we get (7.1), and $k=1$ gives

$$
\gamma_{1}(r, m)=\frac{-1}{m}\left\{\frac{1}{2} \log ^{2} m+(\log m) \psi\left(\frac{r}{m}\right)+\psi_{1}\left(\frac{r}{m}\right)\right\} .
$$

Proof of Proposition 11. We begin with a slight modification of the definition (1.4) and (1.3), valid for $1 \leq r \leq m$,

$$
\gamma_{k}(r, m)=\lim _{n \rightarrow \infty}\left\{\sum_{j=0}^{n} \frac{\log ^{k}(j m+r)}{j m+r}-\frac{\log ^{k+1}(n m+r)}{m(k+1)}\right\} .
$$

We use binomial expansions to obtain

$$
\log ^{k}(j m+r)=\sum_{\nu=0}^{k}\left(\begin{array}{l}
k \\
\nu
\end{array}\right) \log ^{k-\nu} m \log ^{\nu}\left(j+\frac{r}{m}\right)
$$

and

$$
\begin{aligned}
\frac{\log ^{k+1}(n m+r)}{k+1} & =\sum_{\nu=0}^{k+1} \frac{1}{k+1}\left(\begin{array}{c}
k+1 \\
\nu
\end{array}\right) \log ^{k+1-\nu} m \log ^{\nu}\left(n+\frac{r}{m}\right) \\
& =\frac{\log ^{k+1} m}{k+1}+\sum_{\nu=0}^{k}\left(\begin{array}{l}
k \\
\nu
\end{array}\right) \log ^{k-\nu} m \frac{\log ^{\nu+1}(n+r / m)}{\nu+1}
\end{aligned}
$$

Hence,

$$
\begin{aligned}
\gamma_{k}(r, m)= & \frac{-\log ^{k+1} m}{m(k+1)}+\frac{1}{m} \sum_{\nu=0}^{k}\left(\begin{array}{l}
k \\
\nu
\end{array}\right)(\log m)^{k-\nu} \\
& \times \lim _{n \rightarrow \infty}\left\{\sum_{j=0}^{m} \frac{\log ^{\nu}(j+r / m)}{j+r / m}-\frac{\log ^{\nu+1}(n+r / m)}{\nu+1}\right\} .
\end{aligned}
$$

On the other hand, we have

$$
\gamma_{\nu}=\lim _{n \rightarrow \infty}\left\{\sum_{j=1}^{n} \frac{\log ^{\nu} j}{j}-\frac{\log ^{\nu+1} n}{\nu+1}\right\} .
$$

We obtain now (7.3) by subtracting (7.7) from (7.6), using (7.2) and the fact that for fixed $z$,

$$
\lim _{n \rightarrow \infty}\left\{\log ^{\nu+1}(n+z)-\log ^{\nu+1} n\right\}=0 .
$$

This last limit can be found by way of a binomial expansion of $\log ^{\nu+1}(n+z)=$ $(\log n+\log (1+z / n))^{\nu+1}$. The proof is now complete. 
Remark. From (7.2) it follows that $\psi_{k}(1)=-\gamma_{k}$. Thus, (7.3) with $r=m$ implies (2.4)

We give now two applications of Proposition 11. The first one is a special case of a result of Ramanujan (see [3, p. 222]).

Corollary 11. For $k \geq 0, m \geq 1$, and $1 \leq r \leq m$ we have

$$
\begin{aligned}
\frac{1}{m} \sum_{r=1}^{m-1} \psi_{k}\left(\frac{r}{m}\right)= & (-1)^{k+1} \frac{\log ^{k+1} m}{k+1}-\left(1-\frac{1}{m}\right) \gamma_{k} \\
& -\sum_{j=1}^{k}\left(\begin{array}{c}
k \\
j
\end{array}\right)(-1)^{j}(\log m)^{j} \gamma_{k-j}
\end{aligned}
$$

and in particular

$$
\begin{gathered}
\sum_{r=1}^{m-1} \psi\left(\frac{r}{m}\right)=-m \log m-(m-1) \gamma \\
\sum_{r=1}^{m-1} \psi_{1}\left(\frac{r}{m}\right)=m \frac{\log ^{2} m}{2}-(m-1) \gamma_{1}+m(\log m) \gamma .
\end{gathered}
$$

Proof. We sum both sides of (7.3) over all $r, 1 \leq r \leq m-1$, and use (2.8) and (2.4). Then

$$
\gamma_{k}=-\frac{\log ^{k+1} m}{k+1}-\frac{1}{m} \sum_{\nu=0}^{k}\left(\begin{array}{l}
k \\
\nu
\end{array}\right)(\log m)^{k-\nu}\left(\sum_{r=1}^{m-1} \psi_{\nu}\left(\frac{r}{m}\right)-\gamma_{\nu}\right) .
$$

To simplify notation, we set

$$
A_{\nu}:=\sum_{r=1}^{m-1} \psi_{\nu}\left(\frac{r}{m}\right)-\gamma_{\nu}
$$

then

$$
A_{k}=-m \gamma_{k}-m \frac{\log ^{k+1} m}{k+1}-\sum_{\nu=0}^{k-1}\left(\begin{array}{l}
k \\
\nu
\end{array}\right)(\log m)^{k-\nu} A_{\nu} .
$$

We prove now (7.8) by induction. For $k=0$, the relation (7.11) gives (7.9) immediately. Now suppose (7.8) is true up to $k-1$. We substitute $A_{\nu}$ from (7.8) into (7.11) and get

$$
\begin{aligned}
A_{k}= & -m \gamma_{k}-m \frac{\log ^{k+1} m}{k+1}+m S_{k} \\
& +m \sum_{\nu=0}^{k-1}\left(\begin{array}{l}
k \\
\nu
\end{array}\right)(\log m)^{k-\nu} \gamma_{\nu}+m T_{k},
\end{aligned}
$$

where

$$
\begin{aligned}
S_{k} & :=\sum_{\nu=0}^{k-1}\left(\begin{array}{l}
k \\
\nu
\end{array}\right) \frac{(-1)^{\nu}}{\nu+1}(\log m)^{k-\nu}(\log m)^{\nu+1} \\
& =\frac{\log ^{k+1} m}{k+1} \sum_{\nu=0}^{k-1}\left(\begin{array}{l}
k+1 \\
\nu+1
\end{array}\right)(-1)^{\nu}
\end{aligned}
$$


and therefore

$$
S_{k}=\left(1-(-1)^{k}\right) \frac{\log ^{k+1} m}{k+1}
$$

and where

$$
\begin{aligned}
T_{k} & :=\sum_{\nu=0}^{k-1}\left(\begin{array}{l}
k \\
\nu
\end{array}\right)(\log m)^{k-\nu} \sum_{j=1}^{\nu}\left(\begin{array}{l}
\nu \\
j
\end{array}\right)(-1)^{j}(\log m)^{j} \gamma_{\nu-j} \\
& =\sum_{\nu=0}^{k-1} \sum_{j=0}^{\nu-1}\left(\begin{array}{l}
k \\
\nu
\end{array}\right)\left(\begin{array}{l}
\nu \\
j
\end{array}\right)(-1)^{\nu-j}(\log m)^{k-j} \gamma_{j} \\
& =\sum_{j=0}^{k-2}\left(\begin{array}{l}
k \\
j
\end{array}\right)(\log m)^{k-j} \gamma_{j} \sum_{\nu=j+1}^{k-1}\left(\begin{array}{l}
k-j \\
\nu-j
\end{array}\right)(-1)^{\nu-j} .
\end{aligned}
$$

The inner sum in this last expression is

$$
\sum_{\nu=1}^{k-j-1}\left(\begin{array}{c}
k-j \\
\nu
\end{array}\right)(-1)^{\nu}=-1-(-1)^{k-j}
$$

so that

$$
\begin{aligned}
T_{k} & =-\sum_{j=0}^{k-2}\left(\begin{array}{l}
k \\
j
\end{array}\right)(\log m)^{k-j} \gamma_{j}-\sum_{j=0}^{k-2}\left(\begin{array}{l}
k \\
j
\end{array}\right)(-1)^{k-j}(\log m)^{k-j} \gamma_{j} \\
& =-\sum_{j=0}^{k-1}\left(\begin{array}{l}
k \\
j
\end{array}\right)(\log m)^{k-j} \gamma_{j}-\sum_{j=1}^{k}\left(\begin{array}{c}
k \\
j
\end{array}\right)(-1)^{j}(\log m)^{j} \gamma_{k-j}
\end{aligned}
$$

This, with (7.13) and (7.12), proves (7.8).

Remark. If we set $m=2$ in (7.8), we get

$$
\psi_{k}\left(\frac{1}{2}\right)=(-1)^{k+1} 2 \frac{\log ^{k+1} 2}{k+1}-\gamma_{k}-2 \sum_{j=1}^{k}\left(\begin{array}{c}
k \\
j
\end{array}\right)(-1)^{j}(\log 2)^{j} \gamma_{k-j} .
$$

Corollary 12. For $j \geq 0$ and $m \geq 1$, denote

$$
M_{j}(m):=\sum_{d \mid m} \mu\left(\frac{m}{d}\right) d \log ^{j} d .
$$

Then for all $k \geq 0$ and $m \geq 1$ we have

$$
\sum_{\substack{r=1 \\
(r, m)=1}}^{m} \psi_{k}\left(\frac{r}{m}\right)=\frac{(-1)^{k+1}}{k+1} M_{k+1}(m)-\sum_{j=0}^{k}\left(\begin{array}{c}
k \\
j
\end{array}\right)(-1)^{j} M_{j}(m) \gamma_{k-j}
$$

Proof. We sum (7.3) over all $r, 1 \leq r \leq m$, with $(r, m)=1$, multiply both sides by $m$, and equate the left-hand side with the right-hand side of (4.5). If we denote

$$
b_{\nu}:=\sum_{\substack{r=1 \\(r, m)=1}}^{m} \psi_{\nu}\left(\frac{r}{m}\right)
$$


we get

$$
\begin{aligned}
b_{k}= & \frac{1}{k+1}\left\{N_{k+1}(m)-\varphi(m) \log ^{k+1} m\right\} \\
& -\sum_{\nu=0}^{k-1}\left(\begin{array}{l}
k \\
\nu
\end{array}\right)(\log m)^{k-\nu} b_{\nu}-\sum_{\nu=0}^{k}\left(\begin{array}{l}
k \\
\nu
\end{array}\right) N_{k-\nu}(m) \gamma_{\nu} .
\end{aligned}
$$

By an induction similar to the one in the proof of Corollary 11 , we find

$$
\begin{aligned}
b_{k}= & \frac{1}{k+1} \sum_{j=0}^{k+1}\left(\begin{array}{c}
k+1 \\
j
\end{array}\right)(-1)^{j}(\log m)^{j} N_{k+1-j}(m) \\
& -\sum_{j=0}^{k}\left(\begin{array}{c}
k \\
j
\end{array}\right)(-1)^{j} \gamma_{k-j} \sum_{\nu=0}^{j}\left(\begin{array}{l}
j \\
\nu
\end{array}\right)(-1)^{\nu}(\log m)^{j-\nu} N_{\nu}(m) .
\end{aligned}
$$

Using (4.2) and changing the order of summation, we get

$$
\sum_{\nu=0}^{j}\left(\begin{array}{l}
j \\
\nu
\end{array}\right)(-1)^{\nu}(\log m)^{j-\nu} N_{\nu}(m)=M_{j}(m) .
$$

The assertion (7.15) now follows immediately.

Remarks. 1. With $k=0$, and using the facts that $M_{0}(m)=N_{0}(m)$ and $M_{1}(m)=(\log m) N_{0}(m)-N_{1}(m)$, we get with (4.3) and (4.4),

$$
\sum_{\substack{r=1 \\(r, m)=1}}^{m-1} \psi(r, m)=-\varphi(m)\left\{\gamma+\log m+\sum_{p \mid m} \frac{\log p}{p-1}\right\}
$$

this was derived by Lehmer [15].

2. If $m$ is prime, then we have $M_{j}(m)=m \log ^{j} m$ for $j \geq 1$ and $M_{0}(m)=$ $m-1$, and (7.15) coincides with (7.8).

As a final remark in this section, we consider (7.4) and recall that the $\gamma_{1}(r, m)$ can be given explicitly in terms of $\gamma, \gamma_{1}$, and some other known constants for $m=2,3,4$, and 6 (see $\S 6)$. The same is true for the $\psi(r / m)$ (see, e.g., [3, p. 184]). This allows us to give explicit expressions for $\psi_{1}(r / m)$ with $m=2,3,4$, and 6 . These can be considered as properties of the function $R(x)$ defined by Deninger [8].

Example. Let $r=1$ and $m=3$. It is known that

$$
\psi\left(\frac{1}{3}\right)=-\gamma-\frac{3}{2} \log 3-\frac{\pi}{2 \sqrt{3}} .
$$

Using (6.8) and (7.4), we get

$$
\begin{aligned}
\psi_{1}\left(\frac{1}{3}\right)= & -\gamma_{1}+\frac{1}{2}\left(3 \log 3+\frac{\pi}{\sqrt{3}}\right) \gamma+\frac{3}{4} \log ^{2} 3 \\
& +\pi \sqrt{3}\left\{\frac{2}{3} \log 2 \pi-\frac{1}{12} \log 3-\log \Gamma\left(\frac{1}{3}\right)\right\} .
\end{aligned}
$$


Incidentally, with (7.2), we can thus evaluate the series

$$
\sum_{\nu=1}^{\infty}\left\{\frac{\log (\nu+1 / 3)}{\nu+1 / 3}-\frac{\log \nu}{\nu}\right\} \text {. }
$$

Note that the constant $\gamma_{1}$ is cancelled.

Finally, we note that (7.3), considered as a linear system, can be solved for the $\psi_{\nu}(r / m)$ in terms of $\gamma_{k}(r, m)$ (see Corollary 3$)$.

\section{Numerical eVAluation of The $\gamma_{k}(r, m)$}

Lehmer [15] computed the $\gamma_{0}(r, m)$ for $m \leq 9$ by use of the Euler-Maclaurin summation formula. This method can also be used to evaluate the $\gamma_{k}(r, m)$ for $k \geq 1$.

The generalized Euler constants $\gamma_{k}, 1 \leq k \leq 19$, were evaluated by Liang and Todd [16] with the aid of the slightly modified Euler-Maclaurin formula

$$
\begin{aligned}
\lim _{m \rightarrow \infty}\left\{\sum_{j=1}^{m} f(j)-\int_{1}^{m} f(t) d t\right\}= & \sum_{j=1}^{n} f(j)-\int_{1}^{n} f(t) d t-\frac{1}{2} f(n) \\
& -\sum_{j=1}^{M} \frac{B_{2 j}}{(2 j) !} f^{(2 j-1)}(n)+R_{M}(n, \infty)
\end{aligned}
$$

with

$$
R_{M}(p, q)=\frac{1}{(2 M+1) !} \int_{p}^{q} B_{2 M+1}(t-[t]) f^{(2 M+1)}(t) d t,
$$

where $B_{2 M+1}(x)$ is the Bernoulli polynomial of degree $2 M+1$ and $B_{2 j}$ is the $2 j$ th Bernoulli number (see, e.g., $[1$, p. 804]). For $(8.1)$ to hold, we need that $f$ have $2 M+1$ continuous derivatives, and $f^{(\nu)} \rightarrow 0$ as $t \rightarrow \infty$ for $\nu=1,2, \ldots, 2 M+1$. The parameters $n$ and $M$ are to be chosen such that the error $R_{M}(n, \infty)$ stays within prescribed bounds.

It is clear from the definition of the $\gamma_{k}(r, m)$ that for our purposes we have to take

$$
f(t)=\frac{\log ^{k}(m t+r)}{m t+r}, \quad m \geq 1, \quad 1 \leq r \leq m .
$$

The derivatives in (8.1) and (8.2) were computed in [16] by way of a multiple recursion, which would be fairly easy to program. However, we shall use an expression involving Stirling numbers of the first kind $s(n, k)$ (see, e.g., [7, p. $212 \mathrm{ff}$.] or [1, p. 824]).

Lemma 2. Let $f_{k}(x):=\log ^{k} x / x$. Then

$$
f_{k}^{(\nu)}(x)=\frac{k !}{x^{\nu+1}} \sum_{i=0}^{k} s(\nu+1, k+1-i) \frac{\log ^{i} x}{i !} .
$$

Proof. We use the following properties of the Stirling numbers of the first kind:

$$
\begin{gathered}
s(\nu+1, \mu)=0 \text { if } \mu=0 \text { or } \mu>\nu+1 \\
s(\nu+1, \nu+1)=1, \\
s(\nu, \mu)-\nu s(\nu, \mu+1)=s(\nu+1, \mu+1) \text { for } \nu, \mu \geq 0 .
\end{gathered}
$$


We prove now (8.4) by induction over $\nu$. For $\nu=0$, the relation (8.4), with (8.5) and (8.6), gives $f_{k}(x)=\log ^{k} x / x$. Now suppose (8.4) is true for $\nu$. Differentiating both sides, we obtain

$$
\begin{aligned}
f_{k}^{(\nu+1)}(x)=\frac{k !}{x^{\nu+2}}\left\{\sum_{i=1}^{k} s(\nu+1, k+1-i) \frac{\log ^{i-1} x}{(i-1) !}\right. \\
\left.\quad-(\nu+1) \sum_{i=0}^{k} s(\nu+1, k+1-i) \frac{\log ^{i} x}{i !}\right\} \\
=\frac{k !}{x^{\nu+2}} \sum_{i=0}^{k}\{s(\nu+1, k-i)-(\nu+1) s(\nu+1, k+1-i)\} \frac{\log ^{i} x}{i !},
\end{aligned}
$$

where we have used (8.5). We get now (8.4) for $\nu+1$ by applying (8.7).

Remark. In the case $k=1$, we get

$$
f_{1}^{(\nu)}(x)=\frac{(-1)^{\nu} \nu !}{x^{\nu+1}}\left[\log x-\left(1+\frac{1}{2}+\cdots+\frac{1}{\nu}\right)\right]
$$

(see,e.g., [16, p. 171] or [3, p. 197]). This follows from (8.4) with (8.6) and

$$
s(\nu+1,1)=(-1)^{\nu} \nu !, \quad s(\nu+1,2)=(-1)^{\nu+1} \nu !\left(1+\frac{1}{2}+\cdots+\frac{1}{\nu}\right) .
$$

We return now to (8.1). With $f(t)$ as in (8.3), we get

$$
\int_{0}^{n} f(t) d t=\frac{1}{m}\left\{\frac{\log ^{k+1}(m n+r)}{k+1}-\frac{\log ^{k+1} r}{k+1}\right\},
$$

and the left-hand side of (8.1) becomes, with (1.4),

$$
\gamma_{k}(r, m)+\frac{1}{m} \frac{\log ^{k+1} r}{k+1} \text {. }
$$

The second term is cancelled in (8.1); with Lemma 2 we therefore get the first part of the following asymptotic representation.

Proposition 12. For $k \geq 0, m \geq 1$, and $1 \leq r \leq m$ we have

$$
\begin{aligned}
\gamma_{k}(r, m)= & \sum_{j=0}^{n} \frac{\log ^{k}(m j+r)}{m j+r}-\frac{1}{m} \frac{\log ^{k+1}(m n+r)}{k+1}-\frac{1}{2} \frac{\log ^{k}(m n+r)}{m n+r} \\
& -\sum_{j=1}^{M} \frac{k !}{(2 j) !} \frac{B_{2 j}}{m(n+r / m)^{2 j}} \sum_{i=0}^{k} s(2 j, k+1-i) \frac{\log ^{i}(m n+r)}{i !} \\
& +R_{M}(n, \infty) .
\end{aligned}
$$

If $n>e^{k / 7}-1$, then

$$
\left|R_{M}(n, \infty)\right| \leq \frac{2}{1-2^{-2 M}} \frac{(2 M+2) !}{(2 \pi n)^{2 M+1}} \frac{k ! \log ^{k}(m n+r)}{2 M-6} .
$$


Proof. It remains to estimate the error term $R_{M}(n, \infty)$. With $f$ as in (8.3), we have by $(8.4)$

$$
f^{(2 M+1)}(t)=\frac{k ! m^{2 M+1}}{(m t+r)^{2 M+2}} \sum_{i=0}^{k} \frac{1}{i !} s(2 M+2, k+1-i) \log ^{i}(m t+r)
$$

and therefore, using (8.5),

$$
\left|f^{(2 M+1)}(t)\right| \leq k ! m^{2 M+1} \sum_{j=0}^{2 M+2}|s(2 M+2, j)| \frac{\log ^{k}(m t+r)}{(m t+r)^{2 M+2}} .
$$

The sum of the "unsigned" Stirling numbers is $(2 M+2)$ ! (see, e.g., [7, p. 213]). Hence,

$$
\left|f^{(2 M+1)}(t)\right| \leq k !(2 M+2) ! m^{2 M+1} \frac{\log ^{k}(m t+r)}{(m t+r)^{2 M+2}} .
$$

Furthermore, we have

$$
\left|B_{2 M+1}(t-[t])\right|<\frac{1}{1-2^{-2 M}} \frac{2(2 M+1) !}{(2 \pi)^{2 M+1}}
$$

(see, e.g., [1, p. 805]), so that, with (8.10) and (8.2), we get

$$
\left|R_{M}(n, \infty)\right| \leq \frac{2 k ! m^{2 M+1}}{1-2^{-2 M}} \frac{(2 M+2) !}{(2 \pi)^{2 M+1}} \int_{n}^{\infty} \frac{\log ^{k}(m t+r)}{(m t+r)^{2 M+2}} d t
$$

It is easy to verify that $\log ^{k}(m t+r) /(m t+r)^{7}$ is decreasing as a function of $t$ if $\log (m t+r)>k / 7$. Since $t \geq n$ and $m \geq 1, r \geq 1$, this holds when

$$
n>e^{k / 7}-1 \text {. }
$$

Hence, under condition (8.12) we have

$$
\left|R_{M}(n, \infty)\right| \leq \frac{2 k ! m^{2 M+1}}{1-2^{-2 M}} \frac{(2 M+2) !}{(2 \pi)^{2 M+1}} \frac{\log ^{k}(m n+r)}{(m n+r)^{7}} \int_{n}^{\infty}(m t+r)^{-2 M+5} d t
$$

Upon integrating and using the fact that $m n+r>m n$, we obtain the estimate (8.9).

Equation (8.8) proves to be convenient for computation with the symbolic manipulation package MAPLE. Bernoulli and Stirling numbers are "known" to MAPLE; hence minimal programming effort is required. The $\gamma_{k}(r, m)$ were computed for $1 \leq k \leq 20,1 \leq m \leq 9$, and $1 \leq r \leq m$. For $1 \leq k \leq 10$ one obtains, by (8.9), $\left|R_{M}(n, \infty)\right|<0.15 \cdot 10^{-15}$ for $n=40$ and $M=10$. For $11 \leq k \leq 20$, the choice was $n=40$ and $M=20$; in this case, $\left|R_{M}(n, \infty)\right|<$ $0.31 \cdot 10^{-14}$. Because of high cancellations (see the remarks on this problem in [16]), all calculations were carried out to 40 digits accuracy; Table 1 in the Supplement section at the end of this issue shows 12 significant digits. The values for $\gamma_{k}(1,1), 1 \leq k \leq 20$, agree with those for $\gamma_{k}$ in the tables in $[16,4]$. 


\section{BIBLIOGRAPHY}

1. M. Abramowitz and I. A. Stegun, Handbook of mathematical functions, Dover, New York, 1965.

2. T. M. Apostol, Introduction to analytic number theory, Springer-Verlag, New York, 1976.

3. B. C. Berndt, Ramanujan's notebooks, Part I, Springer-Verlag, New York, 1985.

4. J. Bohman and C.-E. Fröberg, The Stieltjes function-definition and properties, Math. Comp. 51 (1988), 281-289.

5. Z. I. Borevich and I. R. Shafarevich, Number theory, Academic Press, New York, 1966.

6. W. E. Briggs, The irrationality of $\gamma$ or of sets of similar constants, K. Norske Vid. Selsk. Forh. (Trondheim) 34 (1961), 25-28.

7. L. Comtet, Advanced combinatorics, Reidel, Dordrecht, 1974.

8. C. Deninger, On the analogue of the formula of Chowla and Selberg for real quadratic fields, J. Reine Angew. Math. 351 (1984), 171-191.

9. K. Dilcher, On a generalized gamma function related to the Laurent coefficients of the Riemann zeta function (in preparation).

10. E. R. Hansen, A table of series and products, Prentice-Hall, Englewood Cliffs, NJ, 1975.

11. M. I. Israilov, On the Laurent expansion of the Riemann zeta-function, Trudy Mat. Inst. Steklov. 158 (1981); English transl., Proc. Steklov Inst. Math. 1983, no. 4, 105-112.

12. E. Jacobsthal, Über die Eulersche Konstante, K. Norske Vid. Selsk. Skrifter (Trondheim), 1967.

13. J. C. Kluyver, On a certain series of Mr. Hardy, Quart. J. Pure Appl. Math. 50 (1927), 185-192.

14. J. Knopfmacher, Generalized Euler constants, Proc. Edinburgh Math. Soc. 21 (1978), 2532.

15. D. H. Lehmer, Euler constants for arithmetical progressions, Acta Arith. 27 (1975), 125-142.

16. J. J. Y. Liang and J. Todd, The Stieltjes constants, J. Res. Nat. Bur. Standards Sect. B 76 (1972), 161-178.

17. Y. Matsuoka, Generalized Euler constants associated with the Riemann zeta function, Number Theory and Combinatorics (Japan 1984), World Scientific, Singapore, 1985, pp. 279295.

18. ___, On the power series coefficients of the Riemann zeta function, Tokyo J. Math. 12 (1989), 49-58.

19. E. P. Stankus, A remark on the coefficients of Laurent series of the Riemann zeta function, Studies in Number Theory, 8, Zap. Nauchn. Sem. Leningrad. Otdel. Mat. Inst. Steklov. (LOMI) 121 (1983), 103-107. (Russian)

Department of Mathematics, Statistics and Computing Science, Dalhousie UniverSity, Halifax, Nova Scotia B3H 3J5, Canada

E-mail address: dilcher@cs.dal.ca 
MATHEMATICS OF COMPUTATION

VOLUME 59, NUMBER 199

JULY 1992, PAGES S21-S24

\title{
Supplement to \\ GENERALIZED EULER CONSTANTS FOR ARITHMETICAL PROGRESSIONS
}

\author{
KARL DILCHER
}
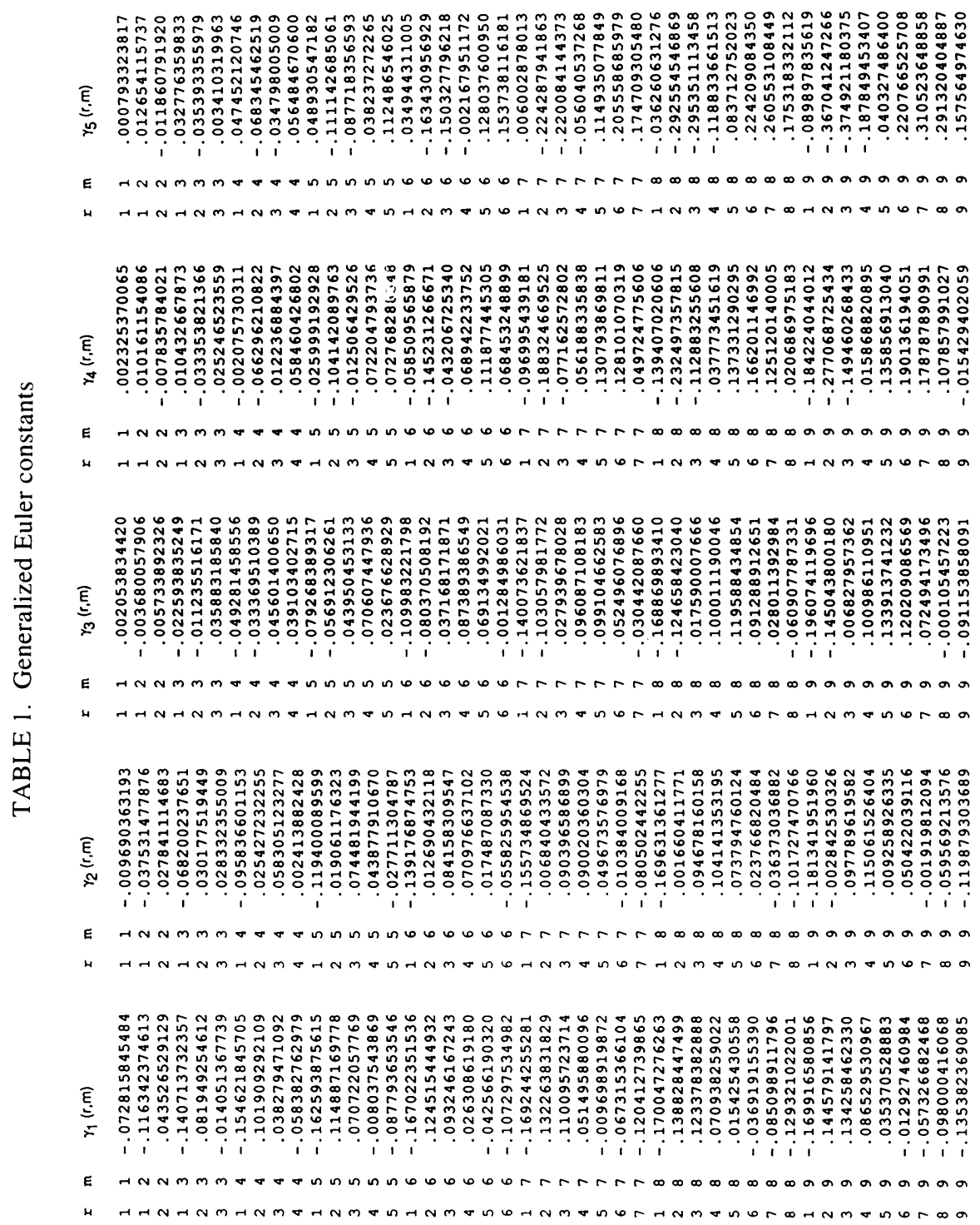

(C) 1992 American Mathematical Society $0025-5718 / 92 \$ 1: 00+\$ .25$ per page 


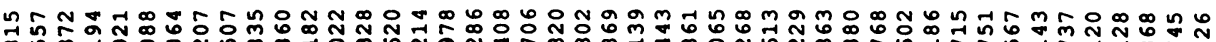

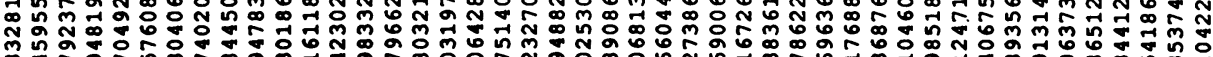
ñ

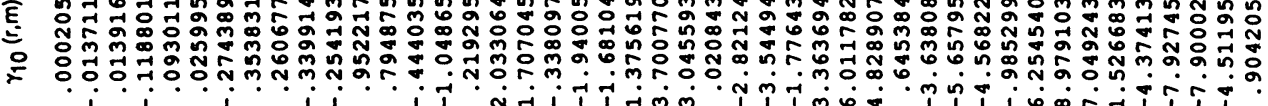
HNNmmmotronmun

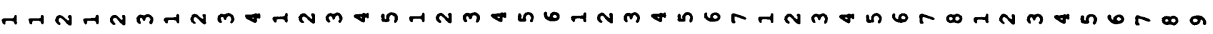

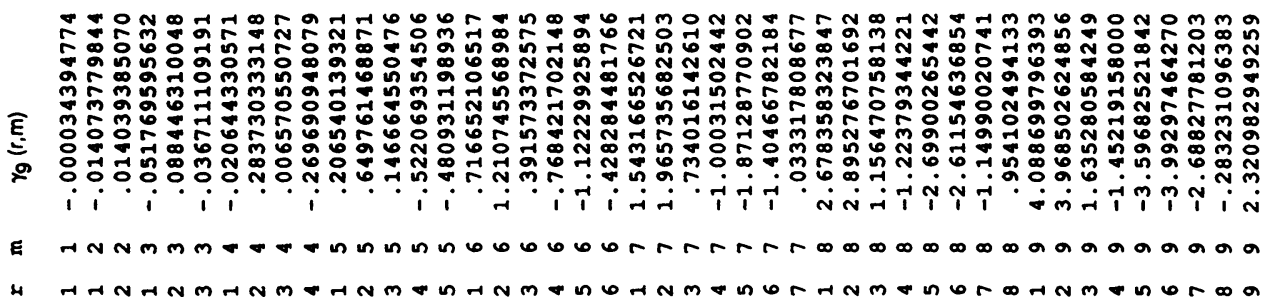

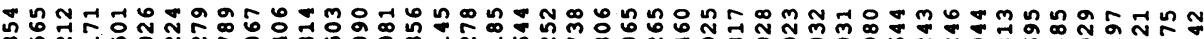

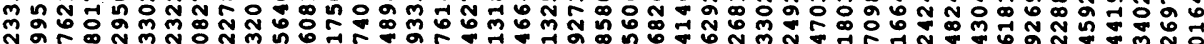

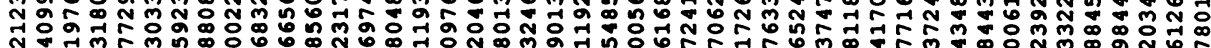

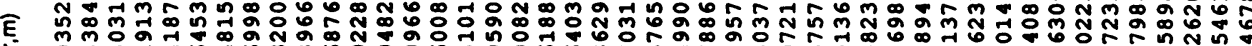
s

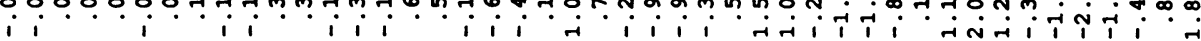

HNNmmmoromen mn

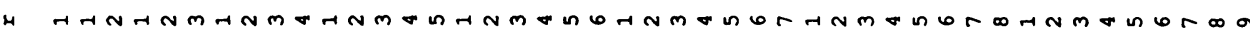
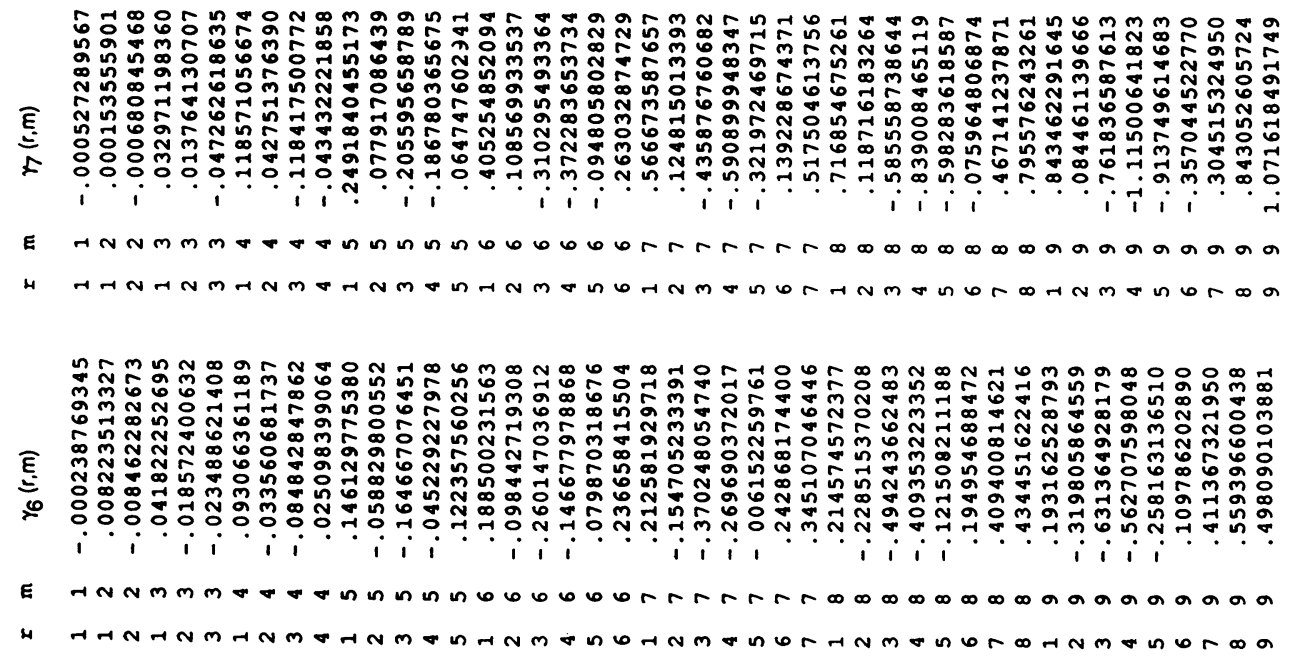


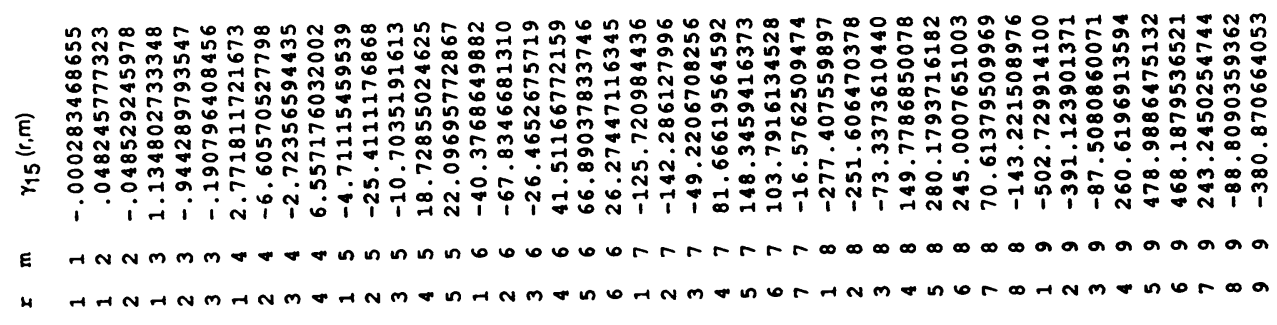

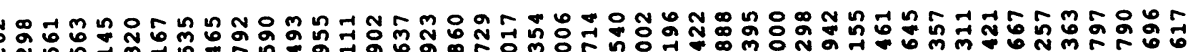

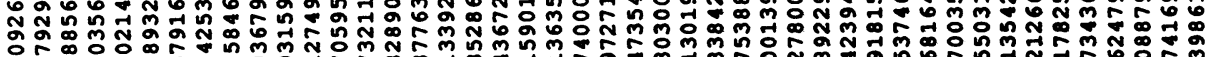

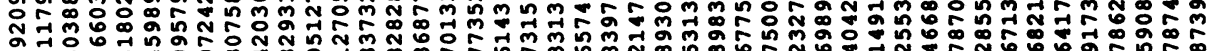
E 士 i

E

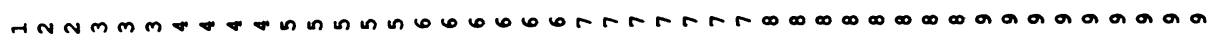

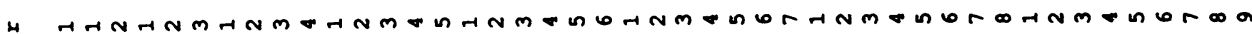

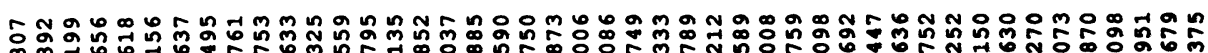

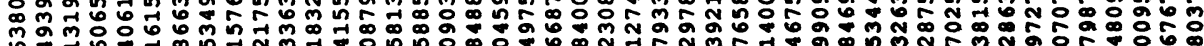

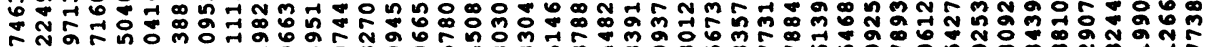

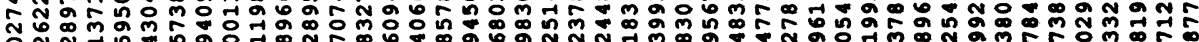

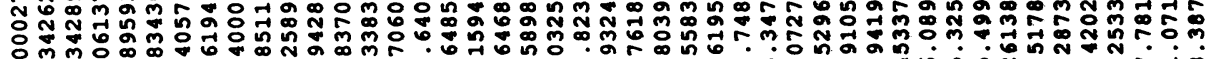

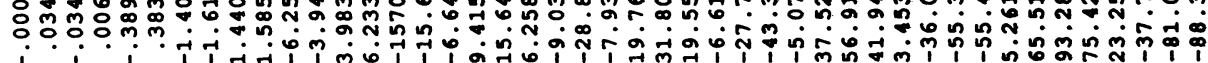
10

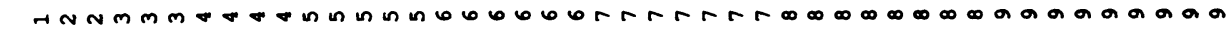

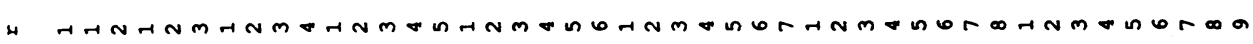

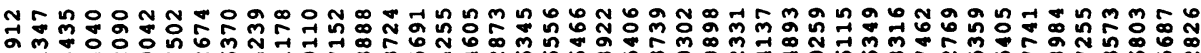

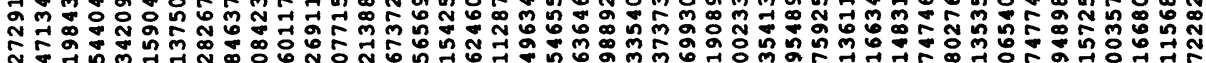

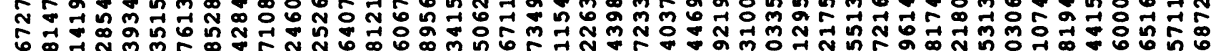

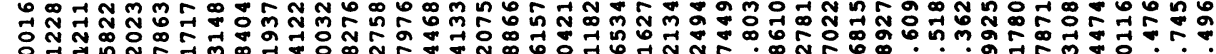

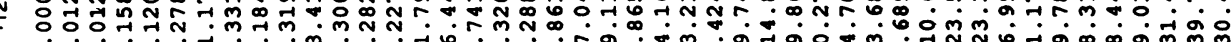

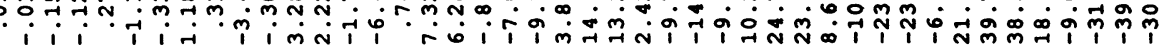

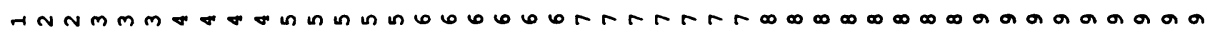

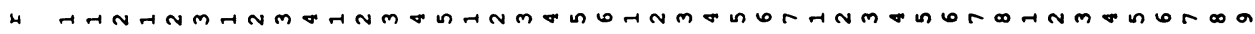

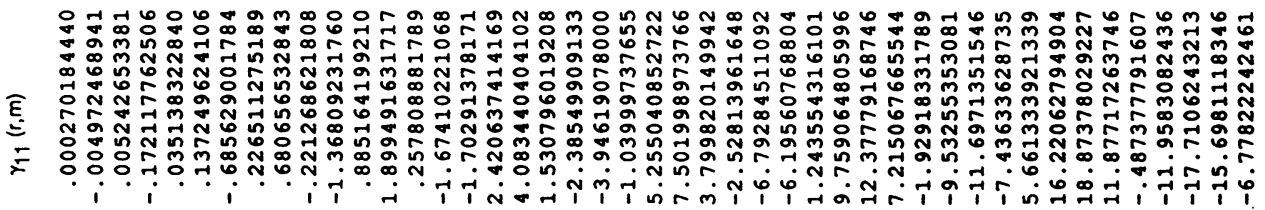

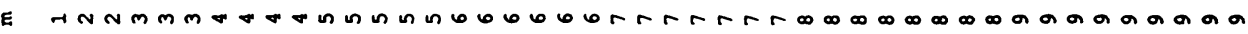

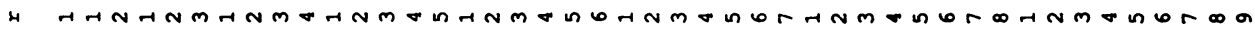




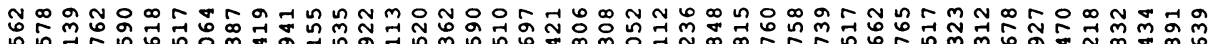

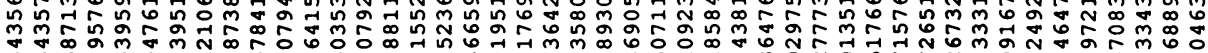

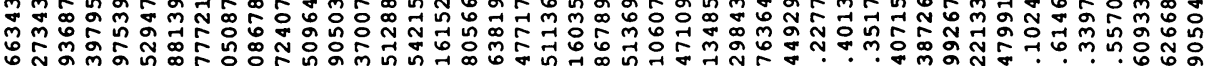

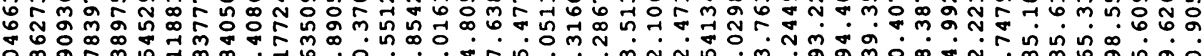

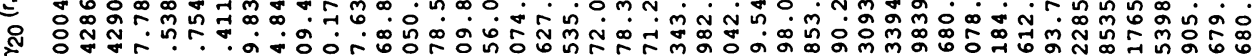

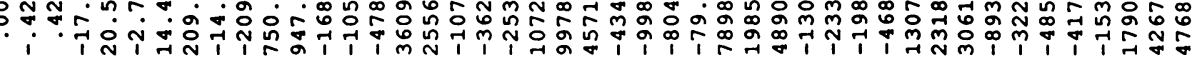

乌ై

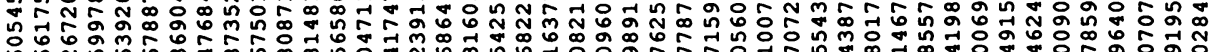

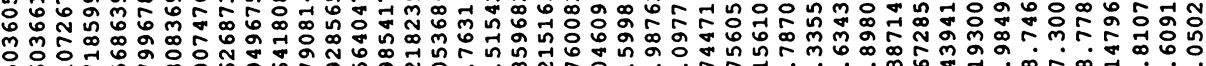

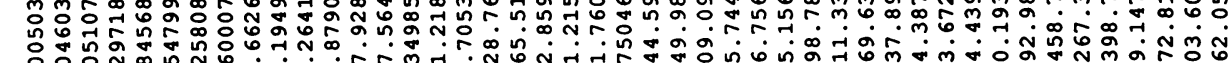

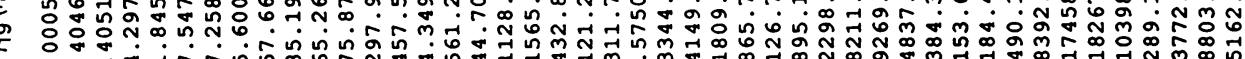

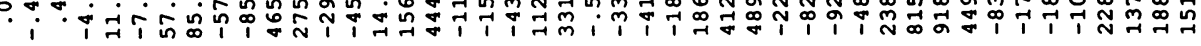

E

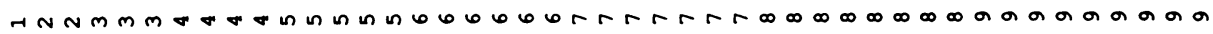

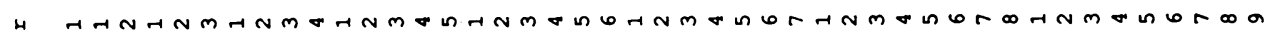

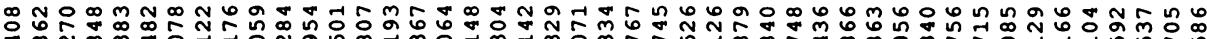

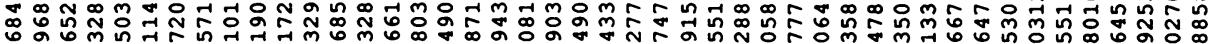
m

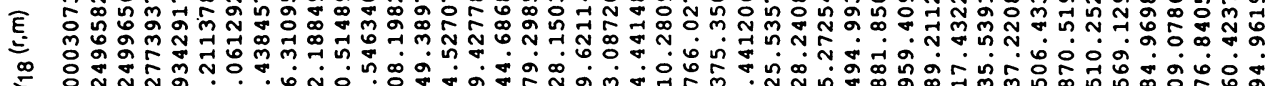

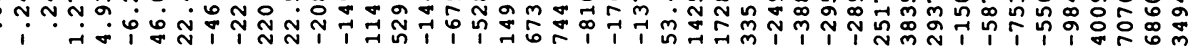

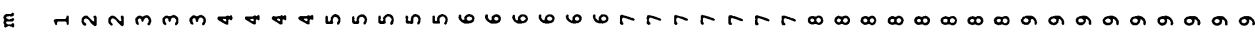

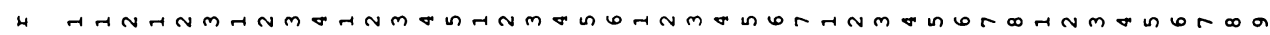

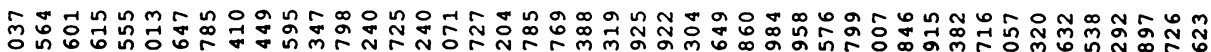

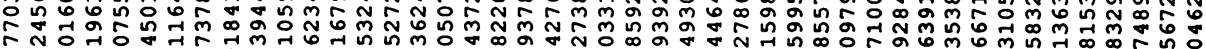

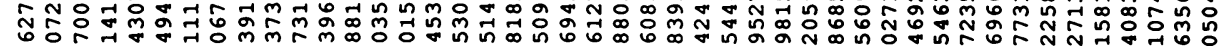

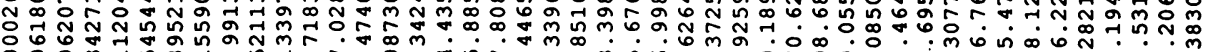

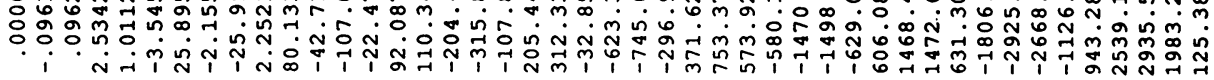

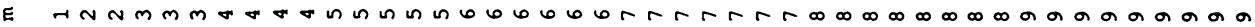

h HAn ham

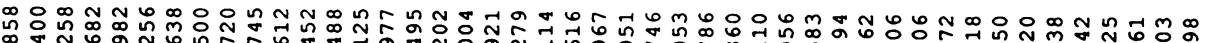

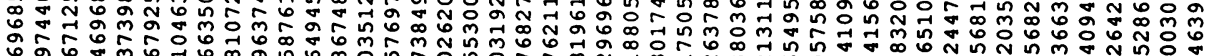

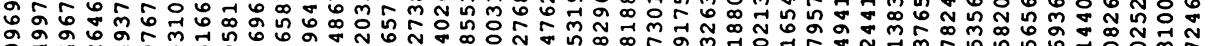
-1
0
0

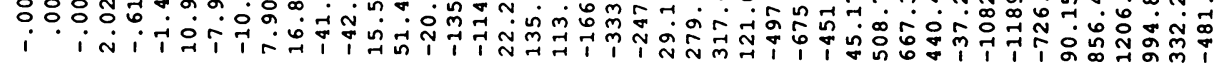

rnNmmmoranon 